\title{
Effects of processing tasks on the recognition of pictures
}

\author{
CLAUDIA J. STANNY and GEORGE F. WEAVER \\ Florida State University, Tallahassee, Florida
}

\begin{abstract}
A levels-of-processing paradigm was used in assessing four different types of study strategy for their importance in picture recognition. Subjects were instructed to attend to colors, large forms, information from multiple sensory modalities, or specific details in studying the pictures. Subjects in a fifth group used any method they wished in order to remember the pictures. The results indicated that if subjects rely on only one strategy, attending to details is a superior strategy for remembering pictures. However, although subjects in the other processing conditions recognized fewer pictures, they were sufficiently accurate to suggest that information other than specific details can play a substantive role in picture memory.
\end{abstract}

A number of studies concerned with the recognition of serially presented pictures have found a positive relation between recognition accuracy and duration of the interstimulus interval (Proctor, 1983; Tversky \& Sherman, 1975; Weaver, 1974; Weaver \& Stanny, 1978). Recognition accuracy also increases when one picture in a list is preceded by a cue that directs subjects to allocate additional processing to that picture (Stanny \& Weaver, 1980; Weaver \& Stanny, 1978, 1984). Furthermore, recognition improves when a cue follows the critical picture (Graefe \& Watkins, 1980; Watkins \& Graefe, 1981).

These results have been interpreted as the consequences of a process analogous to verbal rehearsal. Like verbal rehearsal, the processing given pictures appears to be under voluntary control. However, this process differs from its verbal analog in that it does not generally give rise to serial position effects (Stanny, 1981; Stanny \& Weaver, 1980; Weaver \& Stanny, 1978). Furthermore, although concurrent verbal rehearsal of a list of words reduces recognition accuracy for pictures, it does not appear to specifically interfere with improved recognition that occurs when pictures are cued and given additional processing (Weaver \& Stanny, 1984). These data indicate that changes in recognition accuracy associated with attentional cues are unlikely to be mediated entirely by retaining verbal descriptions of cued pictures.

Although a number of studies have demonstrated that additionally processing pictures will increase the probability that they will be recognized, very little is known about the nature of the processing of these pictures. However, several studies have suggested ways in which processing pictures might differ from processing words

\footnotetext{
This research was reported at the annual meeting of the Southeastern Psychological Association, Atlanta, March 1981. The authors thank Howard Baker for suggestions on the processing tasks and Robert Stanny for helpful comments on the manuscript. Send requests for reprints to George E. Weaver, Department of Psychology, Florida State University, Tallahassee, FL 32306.
}

for retention. For example, the abundance of information available in pictures has been cited as one explanation for the strikingly higher levels of recognition accuracy obtained with pictures than with verbal stimuli (Shepard, 1967). Some investigators have argued that both specific details and more general types of information ("visuospatial information" or "general familiarity") are important for picture recognition (Graefe \& Watkins, 1980; Intraub, 1980; Loftus \& Bell, 1975; Watkins \& Graefe, 1981). The purpose of the present study was to examine the effect of a number of study strategies on picture recognition. Although a variety of study tasks were used, no assumptions were made about their relative "depth" (see Craik \& Lockhart, 1972). Rather, the effect of a study strategy on recognition accuracy was used to examine the relative importance of different types of information available in pictures for recognition.

\section{METHOD}

\section{Design and Subjects}

A between-subjects design was used in studying five different processing tasks. Two study/test sequences of pictures were used to reduce order effects. One hundred students at Florida State University served as subjects and received partial credit for a course requirement. The subjects were tested in small groups (three to five per group). Twenty subjects participated in each processing condition.

\footnotetext{
Materials and Procedure

The stimuli were color slides of outdoor scenes (e.g., beaches, forests, rivers). Forty pictures were presented sequentially by a Kodak Carousel projector controlled by electronic timers. Stimuli were presented in the center of a $1.5 \times 3.0 \mathrm{~m}$ screen and subtended approximately a $40^{\circ}$ horizontal visual angle. Stimulus duration was $1.5 \mathrm{sec}$, and the interstimulus interval was $10 \mathrm{sec}$.

There were five different study strategies. Each group of subjects was instructed to use a particular study strategy (processing task) to maximize their accuracy in picture recognition. Four of these were designed to orient subjects to attend to colors, large forms, nonvisual sensory information, or specific details. The fifth strategy was a control condition, in which subjects were free to use whatever strategy they would usually employ when trying to remember a picture.
} 
The specific processing tasks were as follows. (1) The subjects rated the pictures on a 7-point scale on the basis of the warmth or coolness of their colors. All colors present in the picture were to be taken into account in this rating. (2) The subjects searched for male and female Freudian symbols (allegedly to take advantage of unconscious processes in memory). This task was designed to induce subjects to encode large forms. For each picture, the subjects indicated which, if any, Freudian symbols they were able to identify. (3) The subjects were instructed to form elaborate codes for pictures by supplementing visual information with information from nonvisual sensory modalities. For example, when studying a beach picture, they might supplement visual information by remembering sounds (surf, gulls), smells (a salt breeze), and/or textures or feelings (sand, warm sun) that might be experienced in the scene depicted. The subjects indicated which additional sensory modalities they used when studying each picture. (4) The subjects identified specific details that might aid later recognition and wrote one- or two-word descriptions of these details. (5) The subjects were instructed to "try to remember" the pictures and to rate them on a 7-point scale for expected ease of recognition.

After all 40 pictures had been presented for study, the last 35 pictures were tested using a two-alternative forced-choice procedure. In each case, the target stimulus was a copy of a picture from the study list; the distractor was selected from the same content area as the target and was visually similar to it. Test pictures were presented simultaneously, side by side on the screen, by two additional Carousel projectors and subtended approximately $30^{\circ}$ of horizontal visual angle each. Targets were assigned randomly to the left or right side of the screen, with the constraint that targets occur in each position equally often. The subjects recorded their choice of the left or right picture as the "old" stimulus. When all subjects had responded, the test pair was removed and the next pair was presented. Test pairs were presented in the same serial order as were the study pictures.

\section{RESULTS AND DISCUSSION}

There were significant differences in picture recognition among the processing tasks $[\mathrm{F}(4,90)=10.40$, Mse $=.03$, probability of a Type I error set at .05$].{ }^{1}$ Computation of epsilon for these data indicated that there was a moderately strong relation between processing task and recognition accuracy $(\epsilon=.53)$. The subjects who rated color warmth were least accurate in recognizing old pictures. They scored a mean of $64 \%$ correct for this condition. Coding in multiple modalities and searching for Freudian symbols produced intermediate levels of recognition accuracy (mean percentages correct were $72 \%$ and $73 \%$, respectively). Encoding specific details and rating pictures for their memorability resulted in the most accurate picture recognition (mean percentages correct were $80 \%$ and $77 \%$, respectively, for these conditions).

A Newman-Keuls test of simple effects indicated that recognition performance in the color task was significantly worse than in any other task. In addition, attending to details was significantly more effective than either identifying forms (Freudian symbols task) or supplemental coding in alternate modalities. However, recognition accuracy in the "remember" condition was intermediate between that associated with attending to details and study tasks directed at more "global" types of information (alternate modalities or symbols tasks) and did not differ significantly from any of these other processing conditions.

Although the subjects in the color condition performed somewhat better than chance, this strategy used in isolation appeared to be of little value in remembering and identifying pictures. In contrast, attending to specific details was clearly a valuable strategy for remembering pictures. However, encoding details was not the only method that resulted in accurate picture recognition. Both creating rich, multimodality codes and encoding large forms (symbols task) enabled the subjects to correctly identify a substantial number of old pictures. In fact, percentagecorrect scores in these conditions were not significantly different from those in the "remember" condition.

These data indicate that attending to nondetail information in the "symbols" and "modalities" conditions contributes to the ability to recognize pictures. Admittedly, this information appears to be less useful than specific details. However, if the subjects were in fact using study strategies as instructed, we might speculate that instructions to use some combination of strategies (e.g., both symbols and modalities or modalities and specific details) might improve recognition more than would instructions to encode only specific details.

The recognition tests in this study used targets and distractors that differed primarily in detail. Both types of stimuli were drawn from the same content area and, consequently, were quite similar in color tone. Moreover, targets and distractors frequently contained identical large forms viewed from differing angles and were also likely to give rise to similar associations with other sensory experiences. As Hall (1983) noted, selection of distractors can have a substantial influence on the nature of the results obtained with a recognition test.

Thus, the superiority of detail information might be a consequence of the nature of the lures used in the recognition tests. Unfortunately, this hypothesis will remain untested until pictorial distractors are devised that remain similar to targets in visual detail but that differ from targets in other respects.

\section{REFERENCES}

Craik, F. I. M., \& LockharT, R. S. (1972). Levels of processing: A framework for memory research. Journal of Verbal Learning and Verbal Behavior, 11, 671-684.

Graefe, T. M., \& Watkins, M. J. (1980). Picture rehearsal: An effect of selectively attending to pictures no longer in view. Journal of Experimental Psychology: Human Learning and Memory, 6, 156-162.

HALL, J. F. (1983). Recall versus recognition: A methodological note. Journal of Experimental Psychology: Learning, Memory, and Cognition, 9, 346-349.

INTRAUB, H. (1980). Presentation rate and the representation of briefly glimpsed pictures in memory. Journal of Experimental Psychology: Human Learning and Memory, 6, 1-12.

LofTUS, G. R., \& BELL, S. M. (1975). Two types of information in picture memory. Journal of Experimental Psychology: Human Learning and Memory, 1, 103-113.

Proctor, R. W. (1983). Recognition memory for pictures as a function of poststimulus interval: An empirical clarification of existing literature. Journal of Experimental Psychology: Learning, Memory, and Cognition, 9, 256-262.

SHEPARD, R. N. (1967). Recognition memory for words, sentences, and pictures. Journal of Verbal Learning and Verbal Behavior, 6, 156-163.

STANNY, C. J. (1981). Serial position effects in visual memory: The effects of repetition on item and order information. Unpublished doctoral dissertation, Florida State University, Tallahassee.

Stanny, C. J., \& Weaver, G. E. (1980). Directed attention and the 
recognition of pictures. Bulletin of the Psychonomic Society, 15, 410-412.

TVersky, B., \& Sherman, T. (1975). Picture memory improves with longer on time and off time. Journal of Experimental Psychology: Human Learning and Memory, 1, 114-118.

Watkins, M. J., \& Graefe, T. M. (1981). Delayed rehearsal of pictures. Journal of Verbal Learning and Verbal Behavior, 20, 276-288.

WEAVER, G. E. (1974). Effects of post-stimulus study time on the recognition of pictures. Journal of Experimental Psychology, 103, 799-801.

Weaver, G. E., \& STANNY, C. J. (1978). Short-term retention of pictorial stimuli as assessed by a probe recognition technique. Journal of Experimental Psychology: Human Learning and Memory, 4, 55-65.

Weaver, G. E., \& Stanny, C. J. (1984). The role of detail infor- mation in the recognition of complex pictorial stimuli. Journal of General Psychology, 111, 185-199.

WINER, B. J. (1971). Statistical principles in experimental design (2nd ed.). New York: McGraw-Hill.

\section{NOTE}

1. The arcsin transformation is recommended to stabilize variances when data are proportions (Winer, 1971). The analysis of variance reported was based on arcsin transformed data. Statistical analysis of the untransformed percentages correct yielded identical results.

(Manuscript received for publication December 3, 1984.) 